\title{
A NEW SAMPLE OF THICK DISK AND HALO STARS
}

\author{
C. SOUBIRAN
}

Observatoire de Bordeaux, BP 89, 33270 Floirac, France

M.N. PERRIN AND R. CAYREL

Observatoire de Paris, 75014 Paris, France

\author{
AND \\ E. CHEREUL \\ Observatoire de Strasbourg, 69000 Strasbourg, France
}

The aim of our stellar population study is to investigate the kinematical and chemical characteristics of the thin disk, thick disk and halo of the Galaxy. We have selected 51 stars in 2 astrometric and photometric surveys at $l=42^{\circ}, b=+79^{\circ}$ (Soubiran 1992) and $l=167^{\circ}, b=+47^{\circ}$ (Ojha et al. 1994), on the basis of the Reduced Proper Motion Diagram. They were observed with the $193 \mathrm{~cm}$ telescope at Observatoire de Haute-Provence, with the CARELEC spectrograph (dispersion of $66 \AA / m m, F W H M$ of $3.0 \AA$, range $\lambda \lambda 4600-5500 \AA$ ), together with 43 comparison stars with known fundamental parameters. The derivation of $T_{\text {eff }}, \log g$ and $[\mathrm{Fe} / \mathrm{H}]$ was done differentially using a grid of synthetic spectra and the comparison stars, as described in Cayrel et al. (1991). Twenty of the target stars were found to be more deficient than -0.5 . In the $(\mathrm{V},[\mathrm{Fe} / \mathrm{H}])$ distribution, the halo stars are clearly separated from the other stars with a mean of $(\mathrm{V},[\mathrm{Fe} / \mathrm{H}]) \simeq$ $(-210 \mathrm{~km} / \mathrm{s},-1.4 d e x)$. Because of the small size of the sample, it was not possible to discriminate the thick disk from the thin disk. We have taken 200 more spectra, and with these new observations, we hope to be able to deconvolve the 3 populations in the $(U, V, W,[\mathrm{Fe} / \mathrm{H}])$ space as we did previously with the $(U, V)$ velocity (Soubiran 1993).

\section{References}

Cayrel R., Perrin M.N., Barbuy B., Buser R., 1991, AA 247, 108.

Ojha D.K., Bienaymé O., Robin A.C., Mohan V., 1994, AA 284, 810.

Soubiran C., 1992, AA 259, 394.

Soubiran C., 1993, AA 274, 181. 